\title{
Airy pulses interactions with solitons
}

\author{
$\mathrm{Hao} \mathrm{Wu}$ \\ College of Information Science and Engineering \\ Hunan University \\ Changsha, China \\ e-mail: 865720578@qq.com
}

\author{
Xiquan $\mathrm{Fu}$ \\ College of Information Science and Engineering \\ Hunan University \\ Changsha, China \\ e-mail: fuxiquan@gmail.com
}

\begin{abstract}
We investigate and analyze finite energy Airy pulses interactions with temporal solitons in nonlinear fiber at the same center wavelength, via split step Fourier numerical simulation. The results show that the Airy main lobe is partly trapped by temporal solitons, and the trapped pulse that can present periodic evolution during the propagation is a kind of asymmetric soliton. Although the Airy pulse loses most of the energy to the trapped soliton, the remaining continues to move in the parabolic trajectory. We further study the effect of pump soliton time position and truncation coefficient of Airy pulses on the periodic evolution and energy of trapped soliton.
\end{abstract}

Keywords-finite energy Airy pulses; temporal solitons; interactions; asymmetric soliton; periodic evolution

\section{INTRODUCTION}

Berry and Balazs found that Airy wave packets were a solution to the Schrödinger equation in the context of quantum mechanics in $1979^{[1]}$. However, such ideal Airy beams contain infinite energy, so it is not physically realizeable. In 2007, Siviloglou and Christodoulides demonstrated that truncated Airy beams can exhibit the unique property of acceleration in time ${ }^{[2]}$, as well as ideal Airy beams. These truncated Airy beams can be created by applying a cubic phase mask across a Gaussian beam in the Fourier plane ${ }^{[3]}$. In addition to acceleration, truncated Airy beams also have the unique attributes of self-healing in spite of the severity of the imposed perturbations ${ }^{[4]}$ and resisting dispersion. Since truncated Airy beams have many unique attributes, Airy beams have various applications in different fields such as laser plasma ${ }^{[5,6]}$, particle clearing ${ }^{[7,8]}$, laser filamentation ${ }^{[9]}$. The temporal dispersive equation and the spatial diffraction equation are isomorphic, so truncated Airy pulses have the analogous unique attributes as well as spatial Airy beams. In recent years, truncated Airy pulses are being investigated, in the context of spatiotemporal Airy light bullets in the linear and nonlinear regimes ${ }^{[10]}$, and in the context of soliton shedding from Airy pulses ${ }^{[1]}$, and in the context of supercontinuum generation with femtosecond self-healing Airy pulses ${ }^{[12]}$.

Truncated Airy pulses can propagate a long distance in linear regime in fiber, but eventually succumbing to dispersive. Solitons are the stable solution to nonlinear Schrödinger equation (NLSE), and the result of the balance between dispersion effect and nonlinear effect. Solitons have been extensively studied in both the spatial and temporal domails, the latter out of interest for application in optical communications ${ }^{[13]}$. When weak Airy pulses and strong temporal solitons (the pump) simultaneously propagate in fiber, the pump soliton can generate nonlinear refractive index. Nonlinear refractive index change creates a traveling potential, which can serve as a waveguide for Airy pulses ${ }^{[14,15]}$. So Airy pulses can be trapped by pump solitons. In this paper, we numerically investigate the formation of the trapping of Airy pulses, and we further study the effect of the initial relative time position of pump solitons with Airy pulses and the truncation coefficient of Airy pulses on the periodic evolution and energy of trapped soliton.

\section{AIRY PULSES INTERACTIONS WITH SOLITONS}

\section{A. Theoretical model}

When two optical pulses overlap inside fiber, they can interact with each other through cross-phase modulation (XPM), a phenomenon that is always accompanied by selfphase modulation(SPM). We model Airy pulses interactions with solitons with the normalized coupled $\mathrm{NLSE}^{[16]}$, which neglect the walkoff between Airy pulses and pump solitons, loss of the fiber, and high-order nonlinear effect.

$$
\begin{aligned}
& \frac{\partial U_{1}}{\partial \xi}+\frac{i}{2} \operatorname{sgn}\left(\beta_{21}\right) \frac{\partial^{2} U_{1}}{\partial \tau^{2}}=i N^{2}\left(\left|U_{1}\right|^{2}+2\left|U_{2}\right|^{2}\right) U_{1} \\
& \frac{\partial U_{2}}{\partial \xi}+\frac{\mathrm{i}}{2} \frac{\beta_{22}}{\beta_{21}} \frac{\partial^{2} U_{2}}{\partial \tau^{2}}=\mathrm{i} N^{2}\left(\left|U_{2}\right|^{2}+2\left|U_{1}\right|^{2}\right) U_{2}
\end{aligned}
$$

Where $U_{1}$ and $U_{2}$ are the normalized amplitude of Airy pulses and pump solitons respectively, $\beta_{21}$ and $\beta_{22}$ are the second dispersion coefficient of Airy pulses and pump solitons respectively, $\tau$ and $\xi$ are the retarded time coordinates normalized by the initial width of Airy $\operatorname{pulses}\left(T_{0}\right.$ is the initial width of Airy pulses) and the propagation distance normalized by dispersion length $\left(L_{\mathrm{D}}\right.$ is the dispersion length, $\left.L_{\mathrm{D}}=T_{0}^{2} /\left|\beta_{21}\right|\right)$, the parameter $N^{2}=L_{D} / L_{\mathrm{NL}}\left(L_{\mathrm{NL}}\right.$ is the nonlinear length, $L_{N L}=1 / \gamma_{1} P_{1}$, where $\gamma_{1}$ and $P_{1}$ are the nonlinear coefficient and peak power of Airy pulses).

\section{B. Simulation results}

We demonstrated the trapping of Airy pulses through numerical simulation (1) using the Split Step Fourier Method(SSFM). In simulation, we set $\beta_{21}<0, N=1$. The Airy pulse relative amplitude, phase, and initial time separation impact on the interaction between Airy pulses and solitons ${ }^{[17]}$. Consequently the initial Airy pulses: 
$U_{1}(\xi=0, \tau)=\sqrt{R} \operatorname{Airy}(\tau) \exp (a \tau)$, where $R$ is the peak intensity ratio between Airy pulses and pump solitons, $a$ is the truncation coefficient of Airy pulses. The initial pump solitons: $U_{2}(\xi=0, t)=\operatorname{sech}\left(\tau-\tau_{0}\right) \exp (i \theta)$, where $\tau_{0}$ and $\theta$ are the pump soliton time position and initial phase of pump solitons.

The initial phase $\theta$ and peak intensity ratio $R$ impact barely on the periodic evolution of trapped pulses, so we mainly consider the effect of pump soliton time position $\tau_{0}$ and the truncation coefficient $a$ on the periodic evolution of trapped pulses. In simulation, we choose $\theta=0, R=1 \%$ (ensure that the Airy will propagate in the linear regime), the rang of the pump soliton time position $\tau_{0}=-0.8 \sim 0.8$ (ensure that we distinctly observe the trapping of Airy pulses), and the rang of truncation coefficient $a=0.1 \sim 0.5$ (ensure that we distinctly observe the effect of the truncation coefficient on the periodic evolution of trapped pulses). The simulation results are show in Fig. 1 and Fig. 2. The results show that a relatively stable pulse is formed out of the centered energy about the Airy main labe. The phenomenon illustrates that the Airy main lobe is partly trapped by the pump solitons. The trapped pulses can propagate stably a long distance like the soliton. So we regard the trapped pulses as a kind of special asymmetric soliton. Moreover, the trapped soliton exhibits periodic evolution in the propagation, and the feature of periodic evolution is related with the pump soliton time position and the truncation coefficient of Airy pulses. Although the Airy main lobe loses most of the energy to the trapped soliton, self-healing property of Airy waveform make it recover from the energy loss and the Airy pulse continues to move in parabolic trajectory.
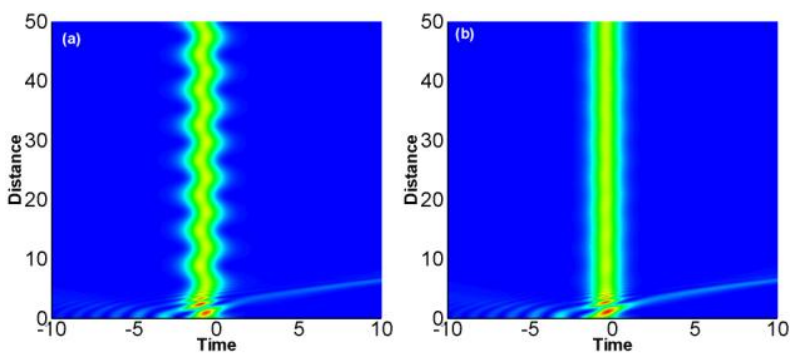

Figure 1. Time-intensity distribution plots of Airy pulses as a function of propagation distance for different pump soliton time position when $a=0.1:$ (a) $\tau_{0}=-0.8$, (b) $\tau_{0}=-0.4$.
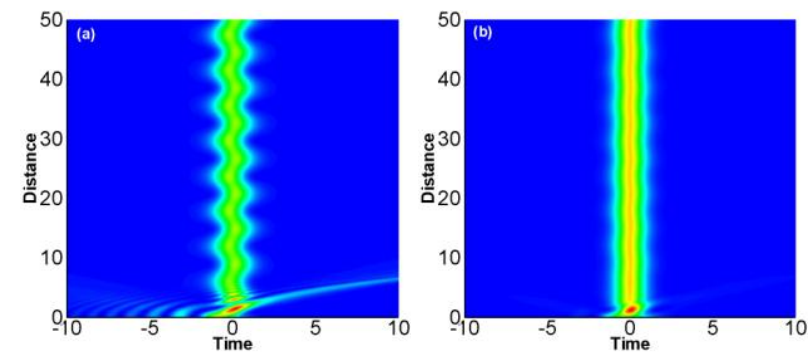

Figure 2. Time-intensity distribution plots of Airy pulses as a function of propagation distance for different truncation coefficient when $\tau_{0}=0$ : (a) $a=0.1$, (c) $a=0.5$.

\section{RESULTS ANALYSIS}

\section{A. The effects of pump soliton time position}

From the Fig. 1, we can see that the features of trapped soliton's periodic evolution are related with the pump soliton time position. In order to investigate the relation between them, we fix the truncation coefficient $a=0.1$ and change the pump soliton time position to observe the central position of trapped soliton (first order moment) as a function of propagation distance. Fig. 3(a) shows a plot of the central position of trapped soliton as a function of propagation distance for different pump soliton time position. The central position of trapped soliton exhibits periodic oscillation, and the pump soliton time position influence the oscillation amplitude and direction, but the oscillation period is invariable. The periodic oscillation is the result of asymmetry of trapped soliton. The oscillation amplitude as a function of pump soliton time position is depicted in Fig. 3(b). With larger pump soliton time position, the oscillation amplitude decreases first and then increases.

Next, we investigate the effect of pump soliton time position on the energy of trapped soliton. Fig. 3(c) shows a plot of the energy of trapped soliton as a function of pump soliton time position. The energy is normalized by the initial energy of Airy main lobe. From the Fig. 3(c), we can see that the energy of trapped soliton decreases with larger pump soliton time position. Because larger pump soliton time position decreases the initial time overlap between the Airy main lobe and pump solitons. Moreover, we find that the most energy of Airy main lobe is trapped by pump solitons, but not completely.
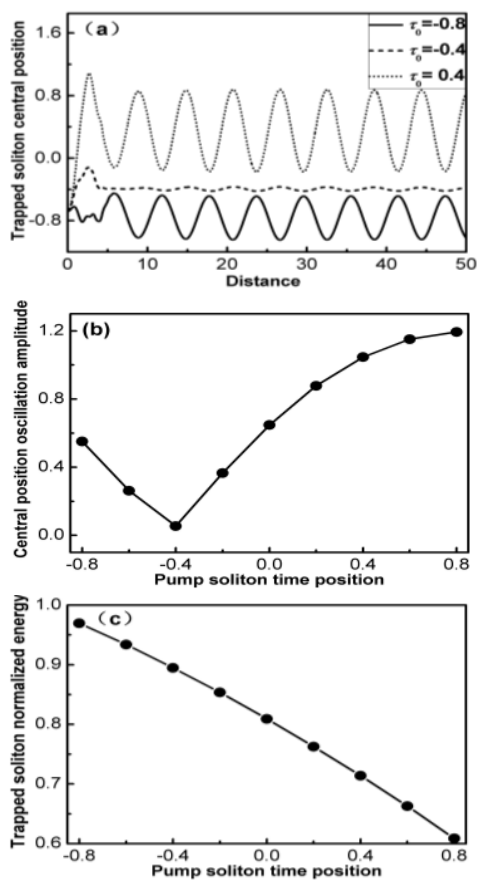

Figure 3. (a) Plots of the central position of trapped soliton as a function of propagation distance for different pump soliton time position when $a=0.1$, (b) Plots of the oscillation amplitude of central position as a function of pump soliton time position when $a=0.1$, (c) Plots of the energy of trapped soliton as a function of pump soliton time position when $a=0.1$. 


\section{B. The effects of truncation coefficient}

From the Fig. 2, we can see that the features of trapped soliton's periodic evolution are related with the truncation coefficient of Airy pulses. In order to investigate the relation between them, we fix the pump soliton time position $\tau_{0}=0$ and change the truncation coefficient to observe the central position of trapped soliton as a function of propagation distance. Fig. 4(a) shows a plot of the central position of trapped soliton as a function of propagation distance for different truncation coefficient. The central position of trapped soliton exhibits periodic oscillation, and truncation coefficient influence the oscillation amplitude, but the oscillation period is invariable. The oscillation amplitude as a function of truncation coefficient is depicted in Fig. 4(b). The oscillation amplitude decreases with larger truncation coefficient, because truncation coefficient influences the peak position of Airy pulses.

Next, we investigate the effect of truncation coefficient on the energy of trapped soliton. Fig. 4(c) shows a plot of the energy of trapped soliton as a function of truncation coefficient. The energy is normalized by the initial energy of corresponding Airy main lobe. As seen in Fig. 4(c), the energy of trapped soliton increases with larger truncation coefficient. Because with the increased truncation coefficient, the Airy main lobe will move right close to pump solitons, which increases the initial time overlap between the Airy main lobe and pump solitons.
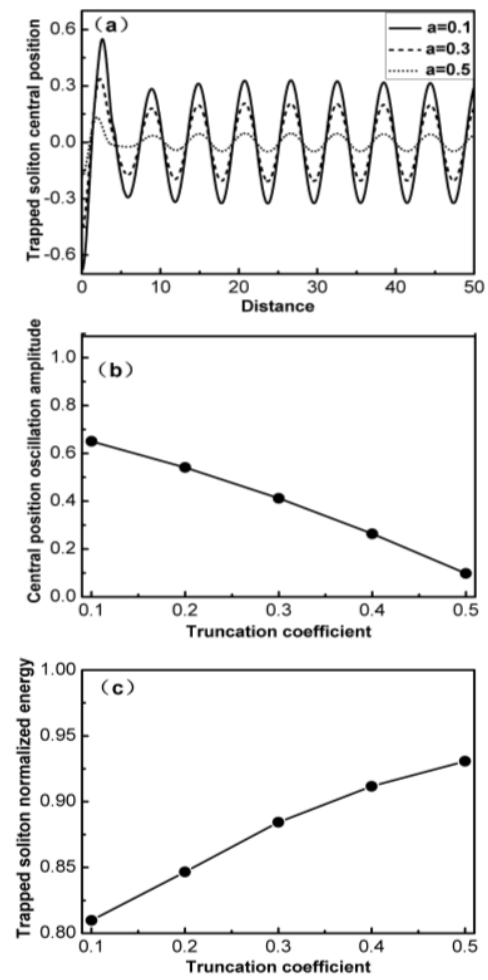

Figure 4. (a) Plots of the central position of trapped soliton as a function of propagation distance for different truncation coefficient when $\tau_{0}=0$, (b)Plots of the oscillation amplitude of trapped soliton's central position as truncation coefficient when $\tau_{0}=0$, (c)Plots of the energy of trapped soliton as a function of truncation coefficient when $\tau_{0}=0$

\section{CONCLUSIONS}

In this paper we investigated finite energy Airy pulses nonlinear interactions with pump solitons in fiber. In simulation, we found that the Airy main lobe is partly trapped by pump solitons under the influence of XPM. The trapped pulse can propagate stably a long distance like the soliton and present the feature of periodic evolution. We regard the trapped pulse as a kind of special asymmetric soliton. The self-healing property of Airy waveforms make the Airy main lobe reconstruction, and the remaining Airy pulse continues to exhibit the unique property of acceleration in time. The feature of trapped soliton's periodic evolution depends both on the pump soliton time position and truncation coefficient of Airy pulses. The energy of trapped soliton is decided by the initial time overlap between the Airy main lobe and pump soliton. At last, While we performed all our analysis in temporal domain, the similar conclusion can be obtained in spatial domain since the spatial diffraction equation and the temporal dispersive equation are isomorphic.

\section{ACKNOWLEDGMENT}

This research is supported in part by the Specialized Research Fund for the Doctoral Program of Higher Education of China (20110161110012), the Department of Science and Technology of Hunan Province, and Hunan Provincial Natural Science Foundation of China (12JJ7005).

\section{REFERENCES}

[1] M. V. Berry, and N. L. Balazs, "Nonspreading wave packets," Am. J. Phys., vol. 43, pp. 264-267, 1979.

[2] G. A. Siviloglou, and D. N. Christodoulides, "Accelerating finite energy Airy beams," Opt. Lett., vol. 32, pp. 979-981, 2007.

[3] G. A. Siviloglou, J. Broky, and A. Dogariu, "Observation of accelerating Airy beams," Phys. Rev. Lett., vol. 99, pp. 213901, 2007.

[4] J. Broky, G. A. Siviloglou, and A. Dogariu, "Self-healing properties of optical Airy beams," Opt. Express, vol. 16, pp. 12880-12891, 2008.

[5] P. Polynkin, M. Kolesik, and J. V. Moloney, "Curved plasma channel generation using ultraintense Airy beams," Science, vol 324, pp. 229-232, 2009.

[6] A. Salandrino, and D. N. Christodoulides, "Airy plasmon: a nondiffracting surface wave," Opt. Lett., vol. 35, pp. 2082-2084, 2010 .

[7] J. Baumgartl, M. Mazilu, and K. Dholakia, "Optically mediated particle clearing using Airy wavepackets," Nat. Photonics, vol. 2, pp. 685-678, 2008.

[8] Cheng Hua, Zang Weiping, and Tian Jianguo, "Study on Optical Trapping and Propulsion of Small Particles by Airy Beam," Acta Optical Sinica, vol. 31, pp. s100405, 2011.

[9] P. Polynkin, M. Kolesik, and J. Moloney, "Filamentation of femtosecond laser Airy beams in water," Phys. Rev. Lett., vol. 103, pp. 123902, 2009.

[10] D. Abdollahpour, S. Suntsov, and D. G. Papazoglou, "Spatiotemporal Airy light bullets in the linear and nonlinear regimes," Phys. Rev. Lett., vol. 105, pp. 253901, 2010.

[11] Y. Fattal, A. Rudnick, and D. M. Marom, "Soliton shedding from Airy pulses in Kerr media,” Opt. Express, vol. 19, 17298-17307, 2011 
[12] C. Ament, P. Polynkin, and J. V. Moloney, "Supercontinuum generation with self-healing Airy pulses," Phys. Rev. Lett., vol 107, pp. 243901, 2011.

[13] H. A. Haus and W. S. Wong, "Solitons in optical communications,” Rev. Mod. Phys., Vol. 68, pp. 423-444, 1996.

[14] R. de la Fuente, and A. Barthelemy, "Spatial Soliton-Induced Guiding by Cross-Phase Modulation," IEEE J. Quantum Electron., vol 28, pp. 547-554, 1992.
[15] Ming-feng Shih, Zhigang Chen, and Matthew Mitchell, "Waveguides induced by photorefractive screening soliton," J. Opt Soc. Am. B, vol. 14, pp. 3091-3101, 1997.

[16] G. P. Agrawal, P. L. Baldeck, and R. R. Alfano, "Temporal and spectral effects of cross-phase modulation on copropagating ultrashort pulses in optical fibers," Phys. Rev. A, vol. 40, pp. 5064, 1989.

[17] A. Rudnick, and D. M. Marom, “Airy-soliton interactions in Kerr media,” Opt. Express, vol. 19, pp. 25570-25582, 2011 